\title{
EFFECT OF SALVAGE MARKET ON STRATEGIC TECHNOLOGY CHOICE AND CAPACITY INVESTMENT DECISION OF FIRM UNDER DEMAND UNCERTAINTY
}

\author{
Mohammad Ali KASHEFI \\ Bielefeld Graduate School of Economics and Management (BiGSEM), \\ Universitätsstrasse 25, D-33615, Bielefeld, Germany \\ E-mail: alikashefii@gmail.com
}

Received 12 May 2012; accepted 24 September 2012

\begin{abstract}
This paper examines the effect of salvage market on technology choice and capacity investment decision of two firms that compete on quantity under demand uncertainty. A game theoretic model applies such that firms choose their production technology between two alternatives: flexible versus inflexible production process. Then they decide on the amount of capacity investment: flexible firm makes decision about general and specific components and inflexible firm just about unified component. One stage forward both enter the primary market in which demand is uncertain and play a la Cournot and finally, flexible firm will be able to sell its unsold general components in the secondary market with a deterministic price. Numerical study was employed to observe equilibrium behavior of firms. Findings demonstrate that with symmetric parameterization there is a unique Nash equilibrium in which both firms choose inflexible technology while applying asymmetric parameters has the potential to form two types of equilibrium when both firms choose inflexible technology or only one firm chooses flexible technology. Moreover, it is shown that there is a cost threshold that could shift the equilibria.
\end{abstract}

Keywords: salvage market, modular and unified production process, product postponement, demand uncertainty, investment decision, operation management.

JEL Classification: C61, C72, C88, D21, L13, M11, Y40.

\section{Introduction}

Intensive competition in global market and product-differentiation strategies of firms force the companies to make their investment decisions in more uncertain environment than before. Uncertainty about the size of the market for potential product and the purchasing behavior of consumers affect the strategic technology choice and capacity investment decision of firms. Actually operation managers try to minimize supply-demand mismatches by considering all available options in the competitive context before choosing their production line technology and decide on their capacity investment. On the other hand, in some industries of developing countries there are large demands for unsold components of some industries in developed countries. In fact, developing 
countries could play the role of salvage market for some companies that encounter low demand realization in the competitive market. Supplying residual general components of some products with prices lower than total cost although implies negative numbers in bottom line of financial statements of a company has the potential of covering some greater loss. Consequently, investment on a modular production line that can further assemble a general and specific component of the final product create the opportunity to respond to the probable demand for unsold components in secondary market. Moreover it can equip the firm with a production technology to hedge against demand uncertainty. Obviously, firm should pay more for extra desirability.

In this paper we explore how the existence of a secondary non-sale capacity market (which we call it salvage market) for unsold general components of a producer affects its strategic technology choice and respected capacity investment decision considering demand variability in the primary market. Our point of departure is the Goyal and Netessine (2007) three-stage model of technology, capacity investment and production games. They show that how a monopolist and duopolist respond to a given flexibility premium. Moreover, in contrast with common belief, they conclude that flexibility is not always the best response to competition such that flexible and dedicated technologies may coexist in equilibrium. They consider two firms that invest in two products and compete with each other in two markets. We introduce salvage market with specific characteristics to their model in which the flexible firm who invests in more expensive technology is able to resell its residual general components with loss. Indeed, we focus on the strategic decisions of two producers upon choosing modular versus unified production line. Modular production line (flexible technology) is designed to assemble general and specific components with higher total cost but can be used as strategic weapon in the presence of demand uncertainty by postponing the production process. On the other hand, unified production line (inflexible or dedicated technology) manufactures the final product without any assembly phase with lower total cost and can be used as commitment device for the producer which ensures the customers of receiving certain amount of goods regardless of the demand realization in the primary market. Furthermore, flexible firm will be able afterwards to enter the salvage market reselling its residual general components with loss, the advantage that does not exist for inflexible producer.

In order to solve the model we have been obliged to apply numerical approach because of intractability of our final equations and integrals. Moreover, uniform distribution function is assumed for handling our demand uncertainty. Under symmetric parameterization we demonstrate that there is a unique symmetric Nash equilibrium such that both producers decide on choosing inflexible (or dedicated) technology and produce the final product via unified production process. In addition, optimal capacity and profits of firms are strictly increasing in mean and standard deviation of the demand intercepts. Under asymmetric parameterization we reach two types of equilibria such that whether both firms choose inflexible technology or just one firm chooses the flexible technology. There is a threshold unified cost around which equilibrium can shift. Disequilibrium also can emerge under some range of parameterization such that we show equilibrium 
in pure strategies for capacity investment fails to exist if the degree of demand variability exceeds a threshold level. The point is that this range of parameters is far from real-world business considerations.

This paper contributes to the available outstanding literature on manufacturing flexibility and production technology by studying the effect of the existence of a nonsale-capacity market which we call it salvage market (or secondary market $B$ ) on the technology choice and capacity investment decision of firms that compete under demand uncertainty. We think that it is worthwhile to investigate this uncovered area of the literature via a separated study.

The remainder of this paper is organized as follows. In part one we briefly review the available related literature in OM and IO. Section 2 explains the basic general model, and section 3 deals with the methodology of solving our problem. In section 4 we report and discuss the findings of our extensive numerical studies, and finally we conclude the research.

\section{Literature review}

Seminal papers in the field of industrial economics and operation management deal with this subject. Production and pricing postponement strategies of producers with respect to revelation of uncertain demand are at the heart of these researches, some investigate just the monopolistic scenario and others consider duopoly competition.

Chod and Rudi (2005) investigated the effect of resource flexibility and responsive pricing for a monopolist doing business in two markets. By using normal distribution in their paper, they show that capacity investment and respected profit are increasing in demand variability, a result that consistently exists in our competitive setting too. Considering market competition, Anupindi and Jiang (2008) endogenize capacity investment, production and pricing decision in their competitive model and evaluate the interplay between the timing of demand realization and production decision of firms with different capabilities. They also establish the strategic equivalence of price and quantity competitions when firms are flexible. Moreover, in their model they characterize equilibria considering two different kinds of demand uncertainty: additive and multiplicative. In our model we deal with additive shock only.

Anand and Girotra (2007) investigate the strategic perils of delayed differentiation and its effect on consumer surplus and welfare. They demonstrate that in the presence of either entry threat or competition, these strategic effects can diminish the value of delayed differentiation (versus early differentiation). In their model they let the producers to decide on the timing of customization freely considering distribution center (DC). Fine and Pappu (1990) evaluate tactical and strategic usage of flexible manufacturing system (FMS) under market competition. Tactical as it helps firm to respond quickly to variation in demand within a market or to decrease the level of inventory and strategic as it equips the firm with a tool to defend its own market and to enter the markets of its less flexible rival. Actually in their two-firm repeated-game model, flexibility serves as a mechanism to prevent market entry by having the potential power of attacking to the 
competitor's markets (grim strategy). Indeed, they show how the availability of FMS can make firms worse off.

McCabe (2011) in its empirical study evaluates the reliability factors for salvage value of photovoltaic (PV). He expressed that as PV system prices become less expensive, the salvage value can be increasingly important in life cycle economic calculations. $\mathrm{He}$ concludes that there is a healthy resale market for PV modules that should be recognized in project level economic evaluation and as systems costs become lower and lower (because of competition), salvage value has more significant ramifications.

Cachon and Koek (2007) explain how to estimate a salvage value of an unsold order. They pointed a quote that describes the economics of selling fashion ski apparel, as faced by Sport Obermeyer: "units left over at the end of the season were sold at a loss that averaged $8 \%$ of the ... price." They believe that choosing a fixed salvage value is questionable and its pricing depends on the amount of left inventory.

\section{The model}

Consider an economy, in which two firms indexed by $j, i, j=1,2$ and $i \neq j$, is producing a homogenous final product. Both firms are assumed to be risk neutral and maximize their expected profits considering the actions of respected rival. Based on the production process technology a single firm chooses, it will be able to produce the final product via whether the unified process or the modular process.

Choosing unified production process enables a firm to manufacture the final product with lower costs and also can be interpreted as a strategic commitment device whereby a firm commits to bring a certain quantity to market (Anupindi, Jiang 2008). On the other hand, choosing modular production process implies that a firm invests on a more expensive technology which empowers it to manufacture the final product with higher costs by producing a general component - which can be used in other products- assembled sequentially with a specific component which is specialized for certain product based on the demand information of the market.

Following the terminology of Anupindi and Jiang (2008), we assume that the firm invests on unified production process is inflexible $(\mathrm{N})$ and the one chooses the modular process is flexible $(F)$ as well. Also we assume that a firm cannot invest in flexible and inflexible technologies simultaneously.

Flexible firm will be able to postpone its production ex post realization of demand which implies more effective reaction to the volatility of market; so it needs to tradeoff the higher costs of flexibility and its ability to hedge against demand uncertainty. On the other hand, inflexible firm commits to produce a certain amount of final good ex ante revelation of demand.

We consider two separated markets here: Market $A$ and market $B$ in which our firms could compete with each other. Market $A$ is the primary market in which demand is uncertain and regardless of the technology choice of our firms, they compete on the quantity of final output in it. (Cournot duopoly competition) Market $B$ is the secondary market with deterministic demand for the general component of the final product which 
can be produced only by the firm chooses the flexible technology. In fact inflexible firm cannot enter this market. Clearly speaking, there is no demand for the final product or specific component in market $B$. Price is also set beforehand less than unit cost of general component procurement.

This paper contributes to the available outstanding literature on manufacturing flexibility and production technology by studying the effect of the existence of a nonsale-capacity market which we call it salvage market (or secondary market $B$ ) on the technology choice and capacity investment decision of firms that compete under demand uncertainty.

A four-stage game theoretic model is applied such that in the first three stages, our firms play a simultaneous-move non cooperative game with complete information.

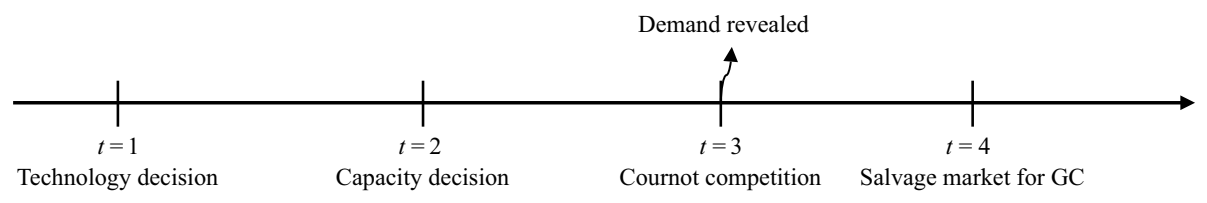

Fig. 1. Four-stage static game

In the first stage $t=1$, each firm can invest either in a flexible technology $(F)$ that enables it to manufacture both general and specific components - which later can be assembled and sold in market $A$ or supplies the general component with known price to market $B$ - or an inflexible technology $(N)$ which allows the firm to produce and supply the final product with lower production costs and higher commitment to market $A$.

Following Goyal and Netessine (2007), three subgames can potentially emerge:

1. Mixed subgame in which one firm invests in flexible and its rival in inflexible technology denoted by $m$. $((F, N)$ or $(N, F))$;

2. Flexible subgame in which both firms invest in flexible technology and have the opportunity to supply the general component in market $\mathrm{B}$, denoted by $f(F, F)$;

3. Inflexible subgame in which both firms choose inflexible technology and the game lasts until the end of the third stage, denoted by $n(N, N)$.

The superscript expresses the subgame which our firm plays denoted by $m, f$ or $n$. Moreover, to differentiate firms from each other, the firm index $i, j$ appears in the subscript as well.

In the second stage $t=2$, each firm invests either in a production capacity of the final product via the unified production process when it adopts inflexible technology or in general and specific components' capacities when it chooses flexible one considering the point that general component can be sold separately in market $B$. Subscripts $g$ and $s$ refer to general and specific components respectively. Moreover, subscript $u$ refers to the final product which is manufactured via unified process.

We denote all capacities by $X$, e.g. $X_{g i}^{m}$ is the capacity of the general component which can be produced by firm $i$ when its rival chooses inflexible technology (Mixed subgame).

Capacity investment is costly and we let these costs to differ by firms. We assume that the cost of purchasing general and specific resources be $c_{g i}$ and $c_{s i}$ per unit respectively 
and the cost of the inflexible resources be $c_{u i}$ per unit for firm $i$. We let the total costs of producing a unit of the final product via the modular process to be $C_{M i}=c_{g i}+c_{s i}$ while for the unified process to be $C_{U i}=c_{u i}$ and so $C_{U i}<C_{M i}$. For the sake of simplicity, we ignore the assembly cost of general and specific component and assume that it is sunk in $c_{g i}$ and $c_{s i}$.

The expected optimal payoff of the firm is denoted by $\Pi$, so e.g. $\Pi_{M i}^{m}$ denotes the expected profit of firm $i$ that compete with firm $j$ in the mixed subgame and invests in two general and specific components via the modular production process technology with capacities $X_{g i}^{m}$ and $X_{s i}^{m}$.

In the third stage $t=3$, firms play a Cournot duopoly game on the quantity of final product they manufacture denoted by $q$. This decision is ex post because at the time of production the firm is better aware of the market demand information.

The linear inverse demand function for the final product which is supplied to market $A$ is $P_{A}\left(A_{A}, Q_{A}\right)=A_{A}-Q_{A}$ in which $Q_{A}=q_{i A}+q_{j A}$ is the total quantity of the final product supplied to the primary market by our firms combined (Cournot competition model with linear demand function) and $P_{A}$ is price of the final product in market $A$ which is assumed to be nonnegative. Subscript $A$ refers to the primary market $A$.

Demand uncertainty appears in the intercepts of the linear inverse demand function, $A_{A} \in \mathfrak{R}_{+}$which draws from a continuous distribution function $F$ with density function $f$. The mean and variance of the marginal distribution is denoted by $\mu_{A}$ and $\sigma_{A}^{2}$ respectively.

We denote profit in the Cournot game by $\pi$ and $E$ represent the expectation operator with respect to the random variable $A_{A}$. Following Goyal and Netessine (2007), marginal cost of production in this stage is normalized to zero. We consider this cost in our capacity decision stage.

Finally in the last stage $t=4$, the firm that has chosen the flexible technology can enter the secondary market $B$ and supplies its unsold general components as a price taker with the deterministic price less than the unit procurement cost of general component which is $P_{B i}<c_{g i}$. Consistent with Roller and Tombak (1990, 1993), modular production process is a prerequisite for entering the secondary market. Figure 2 which is inspired by Anand and Girotra (2007) visually summarizes the explained procedure.

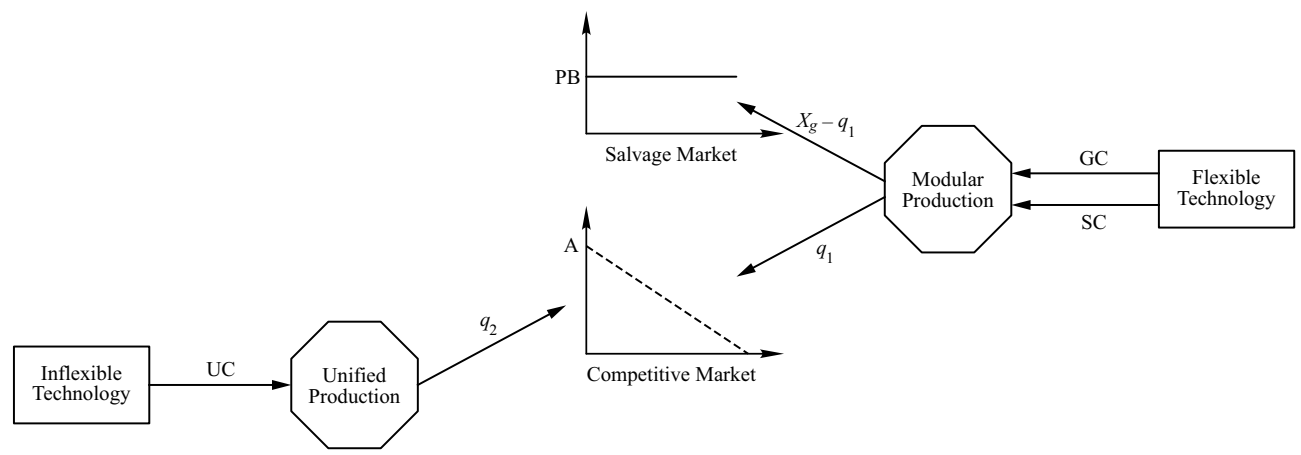

Fig. 2. Modular vs. unified production process 


\subsection{Problem formulation}

Based on the technology choice of our firms which we categorized as three different subgames, this stage could contain zero, one or two player as well. We denote payoff in market $B$ by $v$ which is revenue minus costs there. Following Fine and Pappu (1990) and Roller and Tombak (1990, 1993), we can simply show the technology choice of the firms in a strategic-form game by a $2 \times 2$ matrix as depicted in following page. Matrix entries represent profits in the second-stage capacity game. Backward induction is applied to capture the subgame perfect Nash equilibrium (SPNE) of this model. Hence we move by analyzing from the last stage $t=4$ considering all three possible subgame of the technology choice of our firm. The optimization problem for a firm $i$ that chooses modular production process technology (Flexible firm) for any strategic choice of its competitor $j$ is:

Stage 4: Secondary market for general component

$$
v_{i}=\max _{q_{i B}}\left[q_{i B} . P_{B i}\right] \text { s.t. } 0 \leq q_{i B} \leq\left(X_{g i}-q_{i A}\right) .
$$

Stage 3: Cournot duopoly competition

$$
\pi_{M i}=\max _{q_{i A}}\left[\left(A_{A}-q_{i A}-q_{j A}\right) \cdot q_{i A}+v_{i}\right] \text { s.t. } 0 \leq q_{i A} \leq \min \left[X_{g i}, X_{s i}\right] \text {. }
$$

Stage 2: Capacity decision investment

$$
\Pi_{M i}=\max _{X_{g i}, X_{s i}}\left[E\left(\pi_{M i}\right)-c_{g i} \cdot X_{g i}-c_{s i} \cdot X_{s i}\right] \text { s.t. } X_{g i}, X_{s i} \geq 0 .
$$

The optimization problem for a firm $i$ that chooses unified production process technology (Inflexible firm) for any strategic choice of its competitor $j$ is:

Stage 4: Secondary market for general component $v_{i}=0$.

Stage 3: Cournot duopoly competition

$$
\pi_{U i}=\max _{q_{i A}}\left[\left(A_{A}-q_{i A}-q_{j A}\right) \cdot q_{i A}\right] \text { s.t. } 0 \leq q_{i A} \leq X_{U i} .
$$

Stage 2: Capacity decision investment

$$
\Pi_{U i}=\max _{X_{u i}}\left[E\left(\pi_{U i}\right)-c_{u i} \cdot X_{u i}\right] \text { s.t. } X_{u i} \geq 0 \text {. }
$$

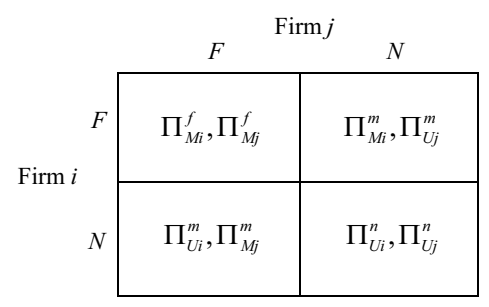

Fig. 3. The strategic-form of the technology game 


\section{Methodology}

In order to solve the model and find the technology choice as well as optimal capacity investment decision of each firm, we proceed by considering each subgame of the model. Backward induction is applied to find the optimal payoff of each probable subgame which afterwards will be located as entries of our mentioned matrix to analyze the equilibria of the model. For the sake of simplicity, we make two assumptions and establish a lemma as follows:

Assumption 1: We assume that both firms enter the game, choose a production technology and make a positive capacity investment which implies that $P\left(A_{A}, 0\right) \geq c_{M}^{\prime}(0)$ for any realization of demand.

Assumption 2: We assume that price is nonnegative for any realization of demand.

Lemma 1: The flexible firm avoids the excess supply of specific components which exists no demand for it in the salvage market $B$ that is $X_{S i} \leq X_{g i}$ or $\min \left[X_{S i}, X_{g i}\right]=X_{S i}$. Based on the model described in previous section, we establish the Lagrangian function of firms in each of mentioned three subgames. Maximization problems are solved using first-order Kuhn-Tucker conditions, but whereas demand is uncertain when firms involve capacity investment decisions, we should consider different states. Each state could happen according to the different probable realization of market size shown by $A$. Hence backward induction approach implies that firms encounter expected profit functions in capacity investment game. Expectation operator leads us to integrals with the boundaries which are functions of capacities and this fact makes our calculation really messy and almost intractable. To simplify the problem we try to specify the probability distribution function of our random variable which appears in the intercept of linear inverse demand function and therefore uniform distribution function $F$ with density $f(A)$ is chosen:

$$
f(A)=\left\{\begin{array}{ll}
\frac{1}{M}, & 0 \leq A \leq M \\
0, & \text { Otherwise }
\end{array} .\right.
$$

Also we add a symmetry assumption between both firms on respected costs' and also salvage market price' parameters.

Whereas these assumptions did not reach us to some gentle equations, we employ an extensive numerical study to find out the strategic behavior of our agents. For this purpose, a wide range of plausible parameters' values chosen to represent realistic scenarios from the real-world businesses. These parameters include costs (general and specific component for flexible firm and unified component for inflexible one shown respectively by $c_{g}, c_{s}$ and $c_{u}$ ), price of the residual general component of flexible firm in salvage market notated by $P_{B}$ and finally $M$ that is a finite positive sufficiently large number such that if demand realization were on the upper bound of probability distribution, all capacities are bounded. Here $M$ has an important interpretation which is inherently in the nature of uniform distribution. Actually the mean and variance of uniform distribution simply 
are $\mu=\frac{M}{2}$ and $\sigma^{2}=\frac{M^{2}}{12}$ respectively which means that the mean and variance of the random variable $A$ (Reservation price of the market) is increasing in $M$. For each parameter combination, we calculated the equilibrium under assumed subgames and determined capacities and profits. The numerical study consists of a large amount of instances resulting from every possible combination of the values listed in Table 1:

Table 1. Parameter values used in numerical study

\begin{tabular}{cc}
\hline Parameter & Values \\
\hline Demand distribution & Uniform \\
\hline$M$ & $(3,120)$ \\
\hline$m$ & $\frac{M}{2}$ \\
\hline$\sigma^{2}$ & $\frac{M^{2}}{12}$ \\
\hline$c_{u}$ & $(1,10]$ \\
\hline$c_{g}$ & $(0.75,10]$ \\
\hline$c_{s}$ & $(0.75,10]$ \\
\hline$P_{B}$ & $(0.5,10)$ \\
\hline Parameters' relations: & $P_{B}<c_{g}, c_{u}<c_{g}+c_{s}$ \\
\hline
\end{tabular}

\section{Findings}

The main part of our analysis contains the technology game in which both firms make decision between modular and unified manufacturing process that afterwards affects the capacity investment decision of them. Seminal papers, including Goyal and Netessine (2007) or Chod and Rudi (2005), despite of some differences in modeling, tried to avoid numerical analysis in this phase and therefore imposed some additional assumptions to ease the analytical discussion. For example, Goyal and Netessine (2007) assume that each firm produces to capacity called it clearance. Numerical approach to solve and analyze of this problem considering a specific distribution function is a missing part of literature that we are going to cover here.

In order to preclude any uncovered set of parameters and results, we were obsessive in examining the parameters. For the purpose of having comprehensive results, also we investigate some sets of parameters which exist numerically but could be interpreted hard economically.

For implementing numerical method, first we choose a reference starting point and then apply incremental approach based on the assumed relationship between parameters, also try to investigate extreme values of them. Optimal capacities and respected maximum profits of producers subsequently are put in the matrix of technology game depicted in Figure 3. In this phase probable equilibrium of the game can be found out by comparing some explicit numbers representing the firms' optimal profit. For detailed mathematical steps refer to technical appendix. 


\subsection{Best reply functions}

In this subsection we are going to characterize the best reply functions of our producers in the capacity investment game. Lemmas $2-4$ characterize the best response functions of both firms. Proofs are put in the technical appendix.

Lemma 2: In flexible subgame of the capacity investment game where both firms choose modular production process, optimal capacities are characterized by best response functions as follows:

$$
\begin{aligned}
& -c_{s_{i}}-c_{g_{i}}+0.25 M+1.5 P_{B}-\frac{0.75 P_{B}^{2}}{M}-X_{s i}+ \\
& \frac{P_{B} \cdot X_{s i}}{M}-\frac{2.25 X_{s i}^{2}}{M}+\frac{4 X_{s i} X_{s j}}{M}-\frac{X_{s j}^{2}}{M}=0, \text { for firm } i, \\
& -c_{s_{j}}-c_{g_{j}}+P_{B}-\frac{P_{B} \cdot X_{s i}}{M}-\frac{2 X_{s i}^{2}}{M}+\frac{4 P_{B} X_{s j}}{M}+\frac{4 X_{s i} X_{s j}}{M}-\frac{2 X_{s j}^{2}}{M}=0, \text { for firm } i .
\end{aligned}
$$

Lemma 3: In inflexible subgame of the capacity investment game where both firms choose unified production process, optimal capacities are characterized by best response functions as follows:

$$
\begin{aligned}
& -c_{u_{i}}+\frac{M}{2}-2 X_{u i}+\frac{1.5 X_{u i}^{2}}{M}-X_{u j}+\frac{2 X_{u i} X_{u j}}{M}+\frac{X_{u j}^{2}}{M}=0, \text { for firm } i, \\
& -c_{u_{j}}+\frac{M}{2}-X_{u i}+\frac{X_{u i}^{2}}{2 M}-2 X_{u j}+\frac{2 X_{u i} X_{u j}}{M}+\frac{2 X_{u j}^{2}}{M}=0 \text {, for firm } j .
\end{aligned}
$$

Lemma 4: In mixed subgame of the capacity investment game where one firm chooses modular production process while the competitor chooses unified one, optimal capacities are characterized by best response functions as follows: (without loss of generality we assume firm $i$ is flexible and firm $j$ is inflexible):

$$
\begin{aligned}
& -c_{g_{i}}-c_{s_{i}}+P_{B}+\frac{M}{2}-2 X_{s i}+\frac{6 P_{B} X_{s i}}{M}+\frac{\left(-2 P_{B}-2 X_{s i}\right) \cdot X_{s i}}{M}+\frac{9 X_{s i}^{2}}{2 M}-X_{u j}+ \\
& \frac{2 X_{s i} X_{u j}}{M}+\frac{-P_{B}^{2}-2 P_{B} X_{s i}-X_{s i}^{2}+X_{u j}^{2}}{M}=0, \text { for firm } i, \\
& -c_{u j}+\frac{M}{2}-X_{s i}+\frac{X_{s i}^{2}}{2 M}-2 X_{u j}+\frac{2 X_{s i} X_{u j}}{M}+\frac{2 X_{u j}^{2}}{M}=0, \text { for firm } j .
\end{aligned}
$$

\subsection{Symmetric parameterization}

Here we start our analysis by assuming symmetry in parameters such that both firms face similar cost of capacities in symmetric subgames $(F, F)$ and $(N, N)$. Moreover, in flexible subgame each should sell the rest of their general component in salvage market with a fixed predetermined price $P_{B}$ (see assumption TA.4 in technical appendix). Figure 4 shows the pair of parameters for each producer that is considered as inputs of numerical solution. 


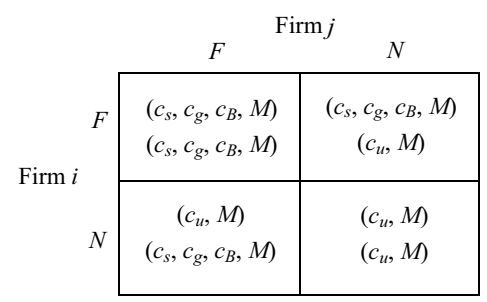

Fig. 4. Symmetric parameterization

Observation 1: Under symmetric parameterization condition, the unique equilibrium of the technology game is the subgame $(N, N)$ that is both firms choose inflexible technology and produce the final product via unified production process. Moreover, this is a symmetric equilibrium such that both choose same amount of capacity investment that is $X_{U i}^{* n}=X_{U j}^{* n}$, which leads to the same optimal profits $\Pi_{U i}^{* n}=\Pi_{U j}^{* n}$.

Observation 2: Optimal capacity and respected profits of firms are strictly increasing in mean $\mu=\frac{M}{2}$ and variance $\sigma^{2}=\frac{M^{2}}{12}$ of the demand intercept and strictly decreasing in the cost of unified component $c_{u}$. (Figs 5 and 6 depict the result for specific amount of parameters.)

Choosing inflexible technology (or unified production process here) can be interpreted as a strategic device whereby a firm commits to bring certain quantity to market. Actu-
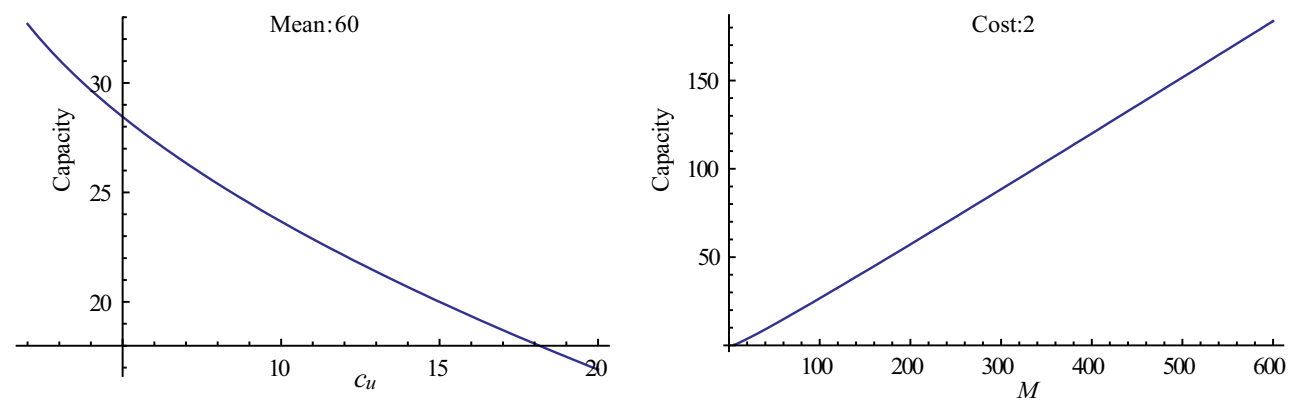

Fig. 5. Optimal capacity investment in inflexible subgame
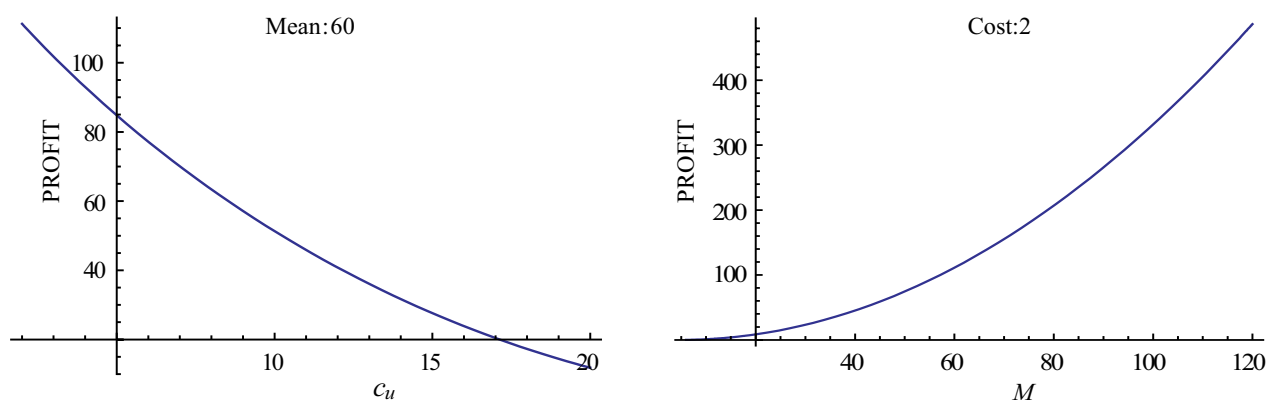

Fig. 6. Optimal profit in inflexible subgame 
ally the firm benefits more from the value of this commitment rather than any flexibility premium it may obtain from the capability to postpone production (Anupindi, Jiang 2008). Our first observation is also consistent with the result of Anupindi and Jiang (2008) that is when $\mu=\frac{M}{2}>c_{u}$ and distribution $F($.) has IGFR (Increasing Generalized Failure Rate) property, which uniform distribution has, there exist unique symmetric equilibrium capacity of a firm in a symmetric inflexible duopoly. The second observation is different from the finding of Goyal and Netessine (2007) that capacity decisions do not depend on variance of demand intercepts. In fact this happens because of the nature of specific probability distribution we choose (Uniform distribution) and also relaxing a tough assumption of that seminal paper that was each firm produces to capacity. The main reason is inherent in the characteristics of uniform distribution such that any change in $M$ causes the simultaneous changes in mean and also variance of demand intercepts (Fig. 7). Although in uniform distribution mean and variance are both the function of one variable, here $M$, but as it is shown in Figure 7, for $M>6$ variance becomes greater than mean and for $M>3$ raises with higher rate than mean. It implies two effects which are happening with increment of $M$ simultaneously: First, an increase in the amount of dispersion escalates the probability of both high and low demand realizations and second, a more attractive mean of market size.

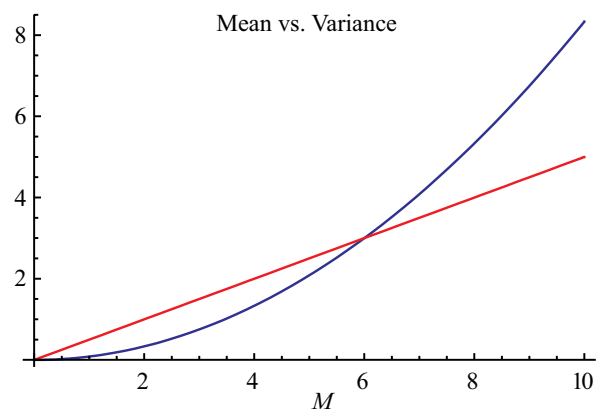

Fig. 7. Mean \& variance of uniform distribution

As it shown in Figure 7, the first effect is stronger for $M>6$ and vice versa. The first effect implies more uncertainty which intuitively might support the usage of flexible technology and the second effect reinforces the investment on inflexible production line in order to commit to a larger market with lower production cost. Furthermore, higher variance and uncertainty spells that for some specific demand realizations, the market clearing price will be zero and so the firm faces some non-sale capacities that in the case of being flexible producer will be able to enter salvage market and sell the general components with loss. Consequently, both firms confront a complex trade-off which has a route in demand uncertainty and cost of producing unified component. Numerical analysis explicitly shows that both firms dominantly prefer to choose inflexible technology and $(N, N)$ is the unique equilibrium of the technology game. Moreover, firms should take into consideration that choosing flexible technology, within this symmetric 
parameterization setting, needs two conditions to be more profitable decision: first, the competitor also should play $F$ and second the firm should invest more rather than its rival on capacity; otherwise you encounter a big loss. Thus playing $F$ has an incredible threat for each manufacturer which leads to the subgame $(N, N)$. Indeed this situation is a kind of prisoner's dilemma game. In the next subsection we run numerical method by considering kinds of asymmetry in some parameters of our established model.

\subsection{Asymmetric parameterization}

Here we relax the assumption of having symmetric parameterization and let our firms obtain their technologies with different investment costs. We can reasonably imagine a case in which both producers having access to similar inflexible technology but they can have different technological level of flexible modular production line. Actually we have implicitly assumed that flexible production strategy is a newer higher technological option that tries to strategically convince stakeholders to invest on it in order to reap more profits from the uncertain demand in the market in comparison with the available inflexible one which is accessible for all firms with same investment cost. Thus in this section we try to scrutinize the scenario that both firms encounter symmetric investment costs when choosing inflexible technology $c_{u i}=c_{u j}$, but asymmetric flexible technological level $c_{g i} \neq c_{g j}$. Figure 8 summarizes the respected parameters' consideration.

Observation 3: Depending on the relative cost of technologies and the upper bound of random variable $M$, it is possible to have two types of equilibrium which is 1 . Both firms are inflexible $(N, N)$ or 2. Only one is flexible $\{(F, N)$ or $(N, F)\}$.

Observation 4: There is a threshold cost of manufacturing the final product via unified production process $c_{u}^{\text {Threshold }}$, after which the firm with access to higher flexible technological capability ( $\operatorname{smaller} c_{M}$ ) finds it more profitable to alter its strategic technology choice from inflexible technology to flexible one which results in asymmetric equilibrium $\{(F, N)$ or $(N, F)\}$.

Observation 5: For sufficiently small amount of $M$ relative to capacity costs, there is a unique Nash equilibrium for this game that is both firms choose inflexible technology $(N, N)$.

Observation 6: For sufficiently large amount of $M$ relative to capacity costs, there is whether a unique Nash equilibrium for this game that is both firms chooses inflexible technology $(N, N)$ or there is no pure strategy Nash equilibrium.

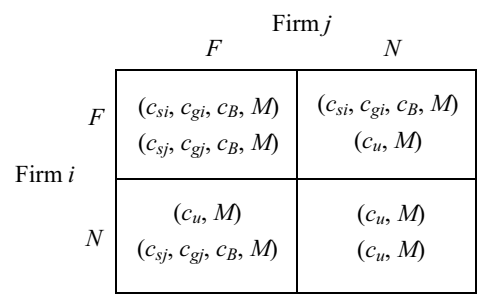

Fig. 8. Asymmetric parameterization 
In this setting two factors actually have significant effects on strategic decisions of our players: first, the perception of producers about the parameter $M$ which implies the maximum possible realization of our random variable $A$ (intercepts of the inverse demand function). It is basically the art of marketing research activities of a company to estimate properly this influential parameter which appears in mean and also variance of the random factor and afterwards affects the strategic decision of firm and also plays role in determination of the amount of capacity investment and respected profits. Second, relative capacity costs of two rival firms which explicitly can change their strategic technology choice. Moreover, as we are working with uniform distribution in this setting, $M$ at the same time clarifies two facts about the market: first, higher $M$ spells more attractive mean of the price reservation. Second, an increase in $M$ increases the likelihood of both high and low demand realizations that is although higher $M$ motivates the producer to take the flexible modular production line but simultaneously increases the threat of higher loss because of very low demand realization and this kind of analysis is reinforced with usage of uniform distribution as we allocate same probability to each level of demand realization. Actually this is the main reason that we face disequilibrium in sufficiently large value of $M$ with respect to capacity costs in some sets of parameters (Observation 6). On the other hand, lower $M$ implies less volatile market which decreases the motivation of investment in more expensive flexible technology such that in sufficient small values of $M$ with respect to capacity costs $(N, N)$ is the unique Nash equilibrium of the game (Observation 5). Consistent with Anupindi and Jiang (2008), we encounter a threshold unified cost - which can be changed with respect to $M$ and modular costs - that whenever $c_{u}<c_{u}^{\text {Threshold }}$, both firms choose inflexible technology and $(N, N)$ is the unique Nash equilibrium of the game, but otherwise the firm with access to higher flexible technological level (lower $c_{M}$ ) finds it more profitable to invest on flexible production line. This results in the formation of asymmetric equilibrium $\{(F, N)$ or $(N, F)\}$ (Observations 3 and 4). Also it should be pointed out that when one manufacturer decides on this strategic move from symmetric inflexible choice to asymmetric flexible one, in some ranges of $M$ it increases the profits of both firms and make them better off. This result depends critically on $M$ such that with higher $M$ the inflexible firm should invest less on capacity and makes less profit in comparison to its flexible rival. Actually higher $M$ causes more marginal benefit for flexible firm which we intuitively expect.

In our setting, as we focus on the effect of salvage market on strategic choice of producers and since the flexible firm is able to sell its unsold general components with predetermined price less than its cost there $P_{B}<c_{g}$, so in our parameterization we have weighted the modular cost with concentration on $c_{g}$ rather than $c_{s}$ and avoided the investigation of extreme scenarios that the main part of the total modular cost exist in specific components such that $c_{s} \gg c_{g}$. In fact, in this case as the revenue of flexible firm in salvage market becomes subtle, there will be no motivation on choosing more expensive modular production line which implicitly bypasses the attraction of our salvage market. Also it can be observed from numerical studies that the most amount of investment on capacities takes place in the symmetric flexible subgame in which both producers rely on their ability to sell their residual general components in salvage market with loss. 
Obviously here the firm that access to higher flexible technology (lower $c_{M}$ ) gets more profit. Although we have assumed that our firms are risk neutral this behavior shows a level of risk taking that is firms hope to face high demand realization in order to obtain more profit. As shown in Figure 6, profit is convex and increasing with respect to demand uncertainty which also reinforce the idea of risk seeking behavior of producers. Moreover, in this case and in the presence of uniform distribution, in higher $M$, risk of facing loss (negative profit) is also high. These are the main reasons that banned the existence of symmetric flexible equilibrium $(F, F)$ as with low $M$ it is not attractive to invest on more expensive less probable modular production technology and in sufficiently large range of $M$ in comparison with inflexible unified technology, it is risky to take flexible technology while the higher standard deviation the larger probability of facing very low demand realization.

\section{Conclusions}

In this paper we present a model to focus on the effect of the existence of a non-sale capacity market (salvage market) on strategic technology choice and capacity investment decision of two firms that compete under stochastic price-dependant demand structure. Actually we take a different approach toward the concepts of flexible production technology and product postponement. Our model is inspired by seminal previous research in this field like Goyal \& Netessine (2007) and Anupindi and Jiang (2008). In this setting each firm involves in three non-cooperative games: technology game (flexible vs. inflexible), capacity investment game (general, specific and unified components) and finally duopoly Cournot game on the amount of quantity. We assumed that flexible firm has the permission to enter the salvage market to ameliorate its excess investment in general components that could occur because of low demand realization. The model is presented in general form, but as it could be followed in technical appendix some simplifying assumptions were essential for solving purposes. Assuming uniform distribution function also did not help us arriving to explicit tractable destination, thus numerical analysis considering broad range of parameters is applied.

We show that depending on the specific values of the problem parameters, three equilibria including $(N, N),(F, N)$ and $(N, F)$ could arise. It was discussed that under symmetric problem parameterization, $(N, N)$ is the unique Nash equilibrium of the game, but in asymmetric setting it is possible to have asymmetric equilibrium in which only one firm chooses flexible technology. In fact, the flexible firm proves the effect of salvage market in strategic-level decision of managers who encouraged by this secondary market to invest on more expensive but better adjusted production line. Moreover, we show in asymmetric case there is a unified cost threshold that can shift the equilibrium of the game. Also the important role of maximum possible market price reservation $M$ is discussed extensively and it is demonstrated that capacity investment and profit of firms are increasing in $M$. Disequilibrium also appears as a result of some specific asymmetric parameterization. Contrary to the common opinion that flexibility is always a competitive advantage against rivals in uncertain markets, it is shown here that the existence of salvage market could convince the managers to employ it just under some specific conditions. 
Several limitations affect the findings of this paper. Uniform distribution is the maximum entropy probability distribution for a random variable that has no constraint except its support interval while in real-world businesses, firms with extensive market research activities has some knowledge about the demand behavior of consumers. Moreover, sufficiently large amount of $M$ under asymmetric problem parameterization eventuate disequilibrium that could restrict the prediction power of our model, even considering the point that large value of $M$ with respect to investment costs implies very high price reservation that within some range of $M$ seems not very logical. Furthermore, setting a fixed price for salvage market is a little bit tough assumption that could be revised in further extension. Development of web-based platforms like eBay, Amazon, or other second hand online markets besides considering large scale salvage markets could be a motivation for further study in this field. Revision the structure of our salvage market, considering two products in primary market, add partial flexibility by letting firms to choose simultaneously flexible and inflexible technologies have the potential of further research.

\section{References}

Anand, K.; Girotra, K. 2007. The strategic perils of delayed differentiation, Management Science 53(5): 697-712. http://dx.doi.org/10.1287/mnsc.1060.0655

Anupindi, R.; Jiang, L. 2008. Capacity investment under postponement strategies, market competition, and demand uncertainty, Management Science 54(11): 1876-1890.

http://dx.doi.org/10.1287/mnsc.1080.0940

Cachon, G.; Koek, A. 2007. Implementation of the newsvendor model with clearance pricing: how to (and how not to) estimate a salvage value, Manufacturing and Service Operations Management 9(3): 276-290. http://dx.doi.org/10.1287/msom.1060.0145

Chod, J.; Rudi, N. 2005. Resource flexibility with responsive pricing, Operation Research 53(3): 532-548. ttp://dx.doi.org/10.1287/opre.1040.0191

Fine, C.; Pappu, S. 1990. Flexible manufacturing technology and product-market competition, Working Paper 3135-90-MSA. Sloan School of Management MIT, Cambridge.

Goyal, M.; Netessine, S. 2007. Strategic technology choice and capacity investment under demand uncertainty, Management Science 53(2): 192-207. http://dx.doi.org/10.1287/mnsc.1060.0611

McCabe, J. 2011. Reliability factors for salvage value of photovoltaic, in Workshop of Photovoltaic Module Reliability, 16-17 February 2011, Golden, Colorado.

Roller, L. H.; Tombak, M. M. 1990. Strategic choice of flexible production technology and welfare implications, Journal of Industrial Economics 38(4): 417-431. http://dx.doi.org/10.2307/2098348

Roller, L. H.; Tombak, M. M. 1993. Competition and investment in flexible technologies, Management Science 39(1): 107-114.

Mohammad Ali KASHEFI. He has obtained his Ph.D. of economics from Bielefeld Graduate School of Economics and Management (BiGSEM) in Bielefeld, Germany. His main field of research is industrial organization and game theory. This paper has done as a part of his doctoral dissertation titled 'Three Essays on Strategic Aspects in Oligopoly with Vertical Structure'. 